\title{
Methylprednisolone or Dexamethasone, Which One Is the Superior Corticosteroid in the Treatment of Hospitalized COVID-19 Patients: A Triple-Blinded Randomized Controlled Trial
}

\section{Keivan Ranjbar}

Shiraz University of Medical Sciences

\section{Reza Shahriarirad}

Shiraz University of Medical Sciences

Amirhossein Erfani

Shiraz University of Medical Sciences

Zohre Khodamoradi

Shiraz University of Medical Sciences

Mohammad Hasan Gholampoor Saadi

Shiraz University of Medical Sciences

Alireza Mirahmadizadeh

Shiraz University of Medical Sciences

Mohammad Javad Fallahi

Shiraz University of Medical Sciences

Vahid Khaloo

Shiraz University of Medical Sciences

Mohsen Moghadami ( $\nabla$ moghadami@sums.ac.ir)

Shiraz University of Medical Sciences

\section{Research Article}

Keywords: Corticosteroid, COVID-19, Dexamethasone, Methylprednisolone, Randomized controlled trial

Posted Date: February 1st, 2021

DOl: https://doi.org/10.21203/rs.3.rs-148529/v1

License: (c) (1) This work is licensed under a Creative Commons Attribution 4.0 International License. Read Full License 
Version of Record: A version of this preprint was published at BMC Infectious Diseases on April 10th, 2021. See the published version at https://doi.org/10.1186/s12879-021-06045-3. 


\section{Abstract}

\section{Background}

Although almost a year has passed since the Coronavirus disease 2019 (COVID-19) outbreak and promising reports of vaccines have been presented, we still have a long way until these measures are available for all. Furthermore, unsolved issues remained to choose appropriate corticosteroids treatment for COVID-19. We conducted a study to assess the effectiveness of methylprednisolone treatment versus dexamethasone for hospitalized COVID-19 patients.

\section{Method}

In this prospective triple-blinded randomized controlled trial, we enrolled 86 hospitalized COVID-19 patients from August to November 2020, in Shiraz, Iran. Patients were randomly allocated into two groups to receive either methylprednisolone $(2 \mathrm{mg} / \mathrm{kg} /$ day) or dexamethasone $(6 \mathrm{mg} / \mathrm{kg} /$ day). Data was assessed based on a 9-point WHO ordinal scale extending from uninfected (point 0 ) to death (point 8).

\section{Results}

There was no significant variation among the groups on the admission. However, the intervention group demonstrated significantly better clinical status compared to the control group at day 5 (4.02 vs. 5.21, $P$ $=0.002)$ and day 10 ( 2.90 vs. $4.71, P=0.001)$ of admission. There was also a significant difference in the overall mean score between the intervention group, and the control group, (3.909 vs. 4.873 respectively, $P$ $=0.004)$. The mean length of hospital stay was $7.43 \pm 3.64$ and $10.52 \pm 5.47$ days in the intervention and control groups, respectively $(P=0.015)$. The need for a ventilator was significantly lower in the intervention group than in the control group $(18.2 \%$ vs $38.1 \% P=0.040)$.

\section{Conclusion}

In the context of treating hospitalized hypoxic COVID-19 patients, methylprednisolone demonstrated better results compared to dexamethasone.

\section{Trial Registration:}

The trial was registered with IRCT.IR (08/04/2020-No. IRCT20200204046369N1).

\section{Background}

Coronavirus disease 2019 (COVID-19) caused by the novel coronavirus, also known as Severe Acute Respiratory Syndrome Coronavirus 2 (SARS-CoV-2), was declared as a global pandemic by WHO on Mar 12,2020 . The disease, causing public health emergency worldwide, has been known to be the third outbreak of beta coronaviruses in the 21st century, after Severe Acute Respiratory Syndrome Coronavirus (SARS-CoV) and Middle East respiratory syndrome coronavirus (MERS-CoV) (1-4). The outbreak was first 
described in December 2019 as a cluster of acute respiratory illnesses in Wuhan, Hubei Province, China, which till January 15, 2021, infected over 93 million cases and caused over 2,000,000 deaths in 218 countries around the world (5). The diseases impact over various aspects has not be unnoticed, from its impact on healthcare system and workers, (6) diagnosis and management dilemmas and overlapping with other disease (7-9), along with its significant mental and emotional impact on the public (10-12).

The relatively high infectivity, rapid progression of lung involvement, and absence of definite effective treatment all contribute to the imperative need to design effective measures of management of COVID-19 based on the disease pathogenesis. Although many types of research and studies have been attributed to the understanding of this disease and various empirical therapeutic options have been introduced on several operational methods, including the existing and new generation of antivirals, steroids, Remdesivir, and traditional medicine, an effective therapeutic option has not yet been achieved mainly for severe COVID-19 cases (13-16).

Earlier studies on SARS showed the overall cytokine dysregulation was the primary pathogenesis of organ dysfunction (17). Therefore, a critical window of opportunity for intervention is considered when status deterioration starts in patients with COVID-19, in which corticosteroids and other immunosuppressive agents can be advantageous, as was the case in experience with SARS and MERS $(18-20)$.

In the United Kingdom, a major randomized clinical trial (RCT) indicated that the use of low-dose dexamethasone in ventilated COVID-19 patients, and to a lesser degree in patients in need of supplemental oxygen, decreased the mortality (21). However, evidence for the intermediate-acting corticosteroid, methylprednisolone, has been limited to date $(22,23)$. In most RCTs, this agent has been used to assist corticosteroids in the intensive care unit (ICU) management of ARDS. Thus, many ICU physicians feel comfortable with administrating this agent (24). Mechanistically, methylprednisolone achieves higher lung tissue-to-plasma ratios in animal models than dexamethasone, which may thus be more effective for lung injury (24). Also, previous studies have shown the effectiveness of methylprednisolone on treating SARS disease $(25,26)$. Hence, we hypothesized that methylprednisolone could be more effective than other corticosteroids, particularly dexamethasone.

Therefore, based on this information, we conducted a randomized control trial to evaluate the effect of methylprednisolone on the outcome of hospitalized COVID-19 patients and compare it with the routinely used dexamethasone according to our national guideline.

\section{Methods}

\section{Patients}

Patients over 18 years that were hospitalized in the main teaching hospital of Shiraz University of Medical Sciences with SARS-CoV-2 infection, which was confirmed by real-time PCR, were enrolled. The inclusion criteria were hospitalized patients above 18 years of age, with an $\mathrm{O}_{2}$ saturation of less than 92 
in room air. The exclusion criteria were pregnancy, uncontrolled diabetes mellitus (DM), uncontrolled hypertension, patients who had previously been treated with steroids for any reason, or any contraindications of steroid administration, immunodeficiency disorders, 02 saturation of above 92 in room air, and lack of willingness to participate in the study.

\section{Study Design}

This study is a stratified triple-blind RCT. Patients were enrolled at Faghihi hospital in Shiraz, Iran, between August 2020 and November 2020, and randomly allocated in a 1:1 ratio to receive a 10-day course of methylprednisolone or dexamethasone with the standard care. Random allocation using the block randomization method was performed in all four branches of the strata, based on two prognostic factors such as age ( $<55$ and $\geq 55$ ) and disease severity based on $\mathrm{O}_{2}$ saturation ( $<85$ and $\geq 85$ ). During the procedure, the allocation remained concealed. The patient, assessor, and analyzer in the two groups did not have access to the randomization list and type of administered drug (Triple blind). All patients received standard care. Furthermore, the intervention group received $2 \mathrm{mg}$ per kilogram of methylprednisolone intravenously daily that was infused over 60 minutes, which was tapered to half dosage every five days. Methylprednisolone treatment was stopped in any patient who faced severe elevations in blood pressure or uncontrolled blood sugar. All patients who were randomized to the control group received $6 \mathrm{mg}$ of dexamethasone intravenously daily for ten days. Figure 1 demonstrates the CONSORT flow diagram of our study.

\section{Clinical and Laboratory Monitoring}

Patient evaluations included demographic features, underlying disease, smoking status, and oxygen saturation, type of oxygen supplementation, respiratory rate, and routine physical exams. To compare the outcome of patients' clinical status in the two groups, a guide provided by the WHO was used called the Ordinal Scale for Clinical Improvement (OSCI), using a 9-point scale, ranging from 0 as no clinical or virological evidence of infection (uninfected) to 8 assigned as death (27). Also, the need for a ventilator during the study, length of hospital stays, and death were noted. The worst score was recorded if the health condition of patients who stayed hospitalized changed on a specific day. On day 10, a final assessment was performed. However, patients were followed for the $28^{\text {th }}$-day outcome in the outpatient clinic after discharge.

\section{End Points}

The primary endpoints were the all-cause mortality in 28 days and clinical status after 5 and 10 days after enrollment with 9-point WHO ordinal scale. If methylprednisolone ought to provide positive results, the distribution of points among patients who received methylprednisolone should swing in the direction of the lower values of the scale compared to the control group.

The secondary endpoints were intubation and need for ventilation, and also admission to ICU. Predetermined exploratory endpoints were the duration of hospital stay and finally, hospital death during 
the 28 days after enrollment. The proportion of patients with these endpoints was also evaluated on days 5 and 10.

\section{Statistics}

Assuming $95 \%$ confidence level (first type alpha error $5 \%$ ) and $80 \%$ power and considering the observation of at least 0.30 and expecting differences in treatment results between the intervention (0.25) and control (0.55) groups, we calculated that a total of 82 COVID-19 patients (i.e. 41 cases in the methylprednisolone group and 41 in the control group) would be required for the analysis (Fleiss with $\mathrm{CC}$ ). Measurement data were described by mean \pm standard deviation (SD) and numeration data were described by number (\%). Statistical differences were assessed using Pearson's chi-square or Fisher's exact tests as categorical variables, as appropriate. The paired-sample t-test was used to evaluate the changes of clinical indexes before and after the administration of methylprednisolone. All analyses were performed in SPSS version 26.0 and $\mathrm{P}<0.05$ was considered statistically significant.

\section{Ethical approval}

The study was approved by the ethics committee of Shiraz University of Medical Sciences (SUMS.REC.1399.014), the institutional review board, and Iranian Registry of Clinical Trials (IRCT20200204046369N1 registered on 08/04/2020) and conducted in compliance with local regulatory requirements, Good Clinical Practice (GCP), and the Declaration of Helsinki (28). Written informed consent was obtained from all patients or their legally authorized representatives.

\section{Results}

A total of 86 patients were enrolled in this clinical trial, in which 44 received methylprednisolone alongside the standard treatment, while 42 received dexamethasone beside the standard treatment and were assigned as the control group. Table 1 demonstrates the baseline data of the patients in our study.

Table 1 Demographic status of subjects in the intervention and control groups at baseline $(\mathrm{N}=86)$. 


\begin{tabular}{|c|c|c|c|c|}
\hline Characteristics & & Intervention $N=44$ & Control $N=42$ & P.Value* \\
\hline \multirow[t]{2}{*}{ Sex } & Male & $27(61.4 \%)$ & $22(52.4 \%)$ & \multirow[t]{2}{*}{0.400} \\
\hline & Female & $17(38.6 \%)$ & $20(47.6 \%)$ & \\
\hline \multirow[t]{6}{*}{ Underlying diseases } & Diabetes & $15(34.1 \%)$ & $13(31.0 \%)$ & 0.756 \\
\hline & Cardiovascular diseases & $12(27.3 \%)$ & $14(33.3 \%)$ & 0.541 \\
\hline & Hypertension & $19(43.2 \%)$ & $20(47.6 \%)$ & 0.679 \\
\hline & Renal diseases & $2(4.5 \%)$ & $0(0 \%)$ & 0.495 \\
\hline & Liver diseases & 0 & 0 & - \\
\hline & Others & $1(2.3 \%)$ & $3(7.1 \%)$ & 0.355 \\
\hline \multirow[t]{3}{*}{ Smoking } & Non-smoker & 27 (61.4\%) & 27 (64.3\%) & \multirow[t]{3}{*}{0.699} \\
\hline & Ex-smoker & $9(20.5 \%)$ & $10(23.8 \%)$ & \\
\hline & Smoker & $8(18.2 \%)$ & $5(11.9 \%)$ & \\
\hline 02 saturation $<85$ & & $22(52.4 \%)$ & $20(48.8 \%)$ & 0.743 \\
\hline Age (year) & & $56.2 \pm 17.5$ & $61.3 \pm 17.3$ & 0.174 \\
\hline
\end{tabular}

* Chi square, Fisher's Exact Test, $T$ test

As demonstrated in Table 1, there was no significant variation among the two groups based on demographic features, comorbid diseases, and disease severity on admission day (02 saturation).

The patients were evaluated at day 0 (on admission), day 5, and day 10 and compared based on OSCl. As demonstrated in Table 2, there was no significant correlation between the OSCl score in the intervention and control groups on admission (4.79 vs. $4.69, \mathrm{P}=0.504)$. However, the intervention group demonstrated significantly lower $\mathrm{OSCl}$ than the control group at day 5 (4.02 vs. $5.21, \mathrm{P}=0.002)$ and day 10 (2.90 vs. $4.71, \mathrm{P}=0.001$ ) of admission.

Table 2 Mean and standard devotion clinical status in the intervention and control groups at days 0, 5 and 10 . 


\begin{tabular}{|c|c|c|c|c|c|}
\hline Time & Group & $\mathbf{N}$ & Mean & Std. Deviation & P.Value* \\
\hline \multirow[t]{2}{*}{ Clinical Status Day 0} & Intervention & 44 & 4.79 & 0.73 & \multirow[t]{2}{*}{0.504} \\
\hline & Control & 42 & 4.69 & 0.71 & \\
\hline \multirow[t]{2}{*}{ Clinical Status Day 5} & Intervention & 44 & 4.02 & 1.64 & \multirow[t]{2}{*}{0.002} \\
\hline & Control & 42 & 5.21 & 1.733 & \\
\hline \multirow[t]{2}{*}{ Clinical Status Day 10} & Intervention & 44 & 2.90 & 2.42 & \multirow[t]{2}{*}{0.001} \\
\hline & Control & 42 & 4.71 & 2.35 & \\
\hline
\end{tabular}

* Independent sample T test

To examine the clinical situation trend on days 0,5 , and 10 , we utilized the repeated measure model. There was a significant effect of time on clinical status, Wilks' Lambda $=0.659, \mathrm{~F}(2,83)=21.450$, $P>0.001$. There was also a significant difference in the overall mean score between the intervention group (3.909 with a range of 3.458-4.360) and the control group (4.873 with a range of 4.411-5.335) $(P=0.004)$.

The repeated ANOVA measurement showed the clinical status score changed significantly during the follow-up for all participants (within-group comparison), $P=0.001$. Also, there was a significant difference after the follow-up between the two groups $(P=0.001)$. (Figure 2$)$

In terms of mortality, the control group reported 15 cases (37.5\%), while in the intervention group, eight patients died (18.6\%); however, there was no significant difference among the two groups $(P=0.076)$.

The hospital stay was also evaluated and compared among the two groups. To make an accurate estimate, we excluded the patients who died during the hospital course. Based on the remaining number of patients, the mean length of hospital stay was $7.43 \pm 3.64$ days and $10.52 \pm 5.47$ days in the intervention and control groups, respectively $(P=0.015)$.

Another outcome indicator was the need to use a ventilator and ICU admission. The need for a ventilator was significantly lower in the intervention group (18.2\%) than in the control group $(38.1 \%)(p=0.040)$

\section{Discussion}

Since the emerge of COVID-19, the world population has faced unprecedented stress. Although almost a year has passed since the outbreak of the disease and promising reports of vaccines have been presented, we still have a long way till these measures are available worldwide. Until that time, the virus continues to obtain many victims and seize many lives, with undesirably high mortality rates among these patients. Thus, physicians have been required to make treatment decisions without substantial evidence during this period. However, since the first reports of the disease in various parts of the world, many data have been gathered and reported to understand the disease characteristics and therapeutic 
management. For instance, reported data have helped the scientific community understand the role of the patients' immune response and its infectious characteristics.

In this study, we aimed to evaluate the therapeutic effect of methylprednisolone as an add-on treatment to the standard treatment regimen of hospitalized COVID-19 patients. Our data were compared with a previously accepted corticosteroid treatment, dexamethasone, based on the hypothesis that methylprednisolone has higher lung penetration $(29,30)$; therefore, it can act as a better immunosuppressive agent in the treatment of COVID-19 and improvement of respiratory complications. Following this theory, our data showed a significant beneficial effect of methylprednisolone in the patients' treatment course and outcome, in terms of clinical status score (based on ordinal scale score), hospitalization duration, and need for mechanical ventilation. Also, the mortality rates were lower in patients who received methylprednisolone than those who received dexamethasone (8 vs. 15). However, this proportion may not be confirmed. Statistically, it was significant in our study and valuable in clinical practice. Such proportions could also achieve statistical significance in a larger population. Another important detail of our research is that we compared methylprednisolone with a previously accepted treatment. In contrast, if compared with patients who do not receive corticosteroids, it goes without saying that methylprednisolone could demonstrate even more optimistic and positive results.

Various observational studies have evaluated the beneficial effects of corticosteroids agents in the treatment of COVID-19 as these agents are widely available, inexpensive, and are easy to use (31-33). Since there had been conflicting results in other viral pneumonia regarding the safety and beneficial effects of corticosteroids, the world health organization (WHO) in the early period of the pandemic published recommendations against the routine use of these agents in managing patients with COVID-19 (26). However, it is well known that glucocorticoid agents are thought to be useful in stopping the inflammatory storm by suppressing pro-inflammatory gene expression and decreasing cytokine levels if used at the appropriate time in the disease course (34). For instance, some studies reported an increase in mortality and prolonged duration of viral clearance using corticosteroids in MERS and Influenza (20, 35). Furthermore, in early studies regarding COVID-19, variations regarding the dosage and administration of corticosteroids have led to inconclusive results about the efficiency of these agents (19). However, later studies have proved the efficacy of methylprednisolone in patients suffering from COVID-19.

In a randomized clinical trial done by Edalatifard et al., the effectiveness of Intravenous methylprednisolone pulse was evaluated (36). In the mentioned study, those who received methylprednisolone had a lower mortality rate and higher survival time than the control group. Moreover, an increase in 02 saturation and BORG scale was observed at the end of the study alongside lesser clinical findings such as myalgia, chest pain, cough, and gastrointestinal symptoms in those who were treated with methylprednisolone compared to those who received standard care. In laboratory findings, the case group experienced a reduction in the CRP level and an increase in the platelet count. Although the dosage and duration of methylprednisolone administration of the mentioned study were different from our research, their results are concomitant. In our study, those who received standard care were 
administered dexamethasone in contrast to the mentioned study above, which also emphasized the superiority of methylprednisolone over administering dexamethasone alone.

In a retrospective cohort study done by Wang et al., evaluating the treatment of patients suffering from COVID-19 with low dose methylprednisolone with short term duration, patients who received 1-

$2 \mathrm{mg} / \mathrm{kg} /$ day methylprednisolone for 5-7 days had shorter hospital course duration, lesser need for mechanical ventilation, but there was no difference in mortality rate compared to those who received standard care, which is in line with our results (37). Further studies also reported a reduction of poor outcomes in patients receiving methylprednisolone (38-40).

In our study, both treated groups received corticosteroids (the control group received dexamethasone); however, those who received methylprednisolone ended up having better outcomes and less dependency on mechanical ventilation. This data suggests that better penetration of methylprednisolone in the lungs compared to dexamethasone results in more reduction in cytokine storm originating from the respiratory system. Thus, a better reduction is seen in the overall respiratory system inflammation compared to dexamethasone. This is in the same line with other studies that demonstrated better penetrance of methylprednisolone in the lung tissue than other corticosteroids (41-43).

Though it should be kept in mind that managing patients suffering from COVID-19 with glucocorticoids may have some complications such as superimposed infection, immunosuppression, and hyperglycemia, recent studies reported no significant complications in their study course. However, hyperglycemia was more frequent in those who received methylprednisolone, managed without substantial complications $(36-38,40)$. Moreover, it is suggested that the full dose of proper antibiotic therapy and immune regulators such as human immunoglobulin should be used to enhance the patients' immunity in cases with complications (37).

This study had several limitations, including the small sample size in each group and limited data regarding the complications, lab data, and computed tomography features. Given the limitations of the study, further randomized controlled trials are needed with larger sample sizes and later follow-ups to evaluate the beneficial effect of methylprednisolone in patients with COVID-19 pneumonia.

\section{Conclusion}

The findings of this clinical trial demonstrated that the administration of intravenous methylprednisolone with a dose of $1-2 \mathrm{mg} / \mathrm{kg}$ in the hospitalized patients suffering from COVID-19 pneumonia and hypoxemia is associated with a reduction in hospitalization period and the need for mechanical ventilation as well as experiencing better clinical status in the hospital; it can be administered as a superior immunosuppressive agent compared to dexamethasone in the treatment of hospitalized COVID19 patients.

\section{Abbreviations}


CONSORT

Consolidated standards of reporting trials; COVID-19:Coronavirus disease 2019; DM:Diabetes mellitus; GCP:Good Clinical Practice; ICU:intensive care unit; MERS-CoV:Middle East respiratory syndrome coronavirus; OSCl:Ordinal Scale for Clinical Improvement; RCT:Randomized controlled trial; SARSCoV:Severe Acute Respiratory Syndrome Coronavirus; SARS-CoV-2:Severe Acute Respiratory Syndrome Coronavirus-2; SD:standard deviation;

\section{Declarations}

\section{Acknowledgments}

The authors would like to thank Shiraz University of Medical Sciences, Shiraz, Iran, and also the Center for Development of Clinical Research of Nemazee Hospital and Dr. Nasrin Shokrpour for editorial assistance. This study was the subject of MD dissertation of Keivan Ranjbar (Thesis No. 22403).

\section{Authors' contributions}

MM, MJF and VK contributed to the conception or design of the work. MHGS, AE and KR contributed to the acquisition and analysis of the data. RS, AM and KR contributed to the interpretation of the data for the work. AE, ZK, RS and KR drafted the work. MM and KR revised it critically for important intellectual content. All authors approved the final version to be published.

\section{Funding}

No specific funding was obtained for this study.

\section{Availability of data and materials}

All de-identified data and statistical codes used to generate the results will be available on request to the corresponding author.

\section{Ethics approval and consent to participate}

This study obtained ethics approval from the Institutional Review Board of Shiraz University of Medical Sciences (SUMS.REC.1399.014). The study was registered on IRCT.IR with the following identifier: IRCT20200204046369N1 on 08/04/2020. Written informed consent was obtained from all patients or their legally authorized representatives.

\section{Consent for publication}

Not applicable

\section{Competing interests}

All authors declare that they have no competing interests. 


\section{References}

1. Eurosurveillance Editorial Team. Note from the editors: World Health Organization declares novel coronavirus (2019-nCoV) sixth public health emergency of international concern. Euro Surveill. 2020;25(5):200131e. doi:10.2807/1560-7917.ES.2020.25.5.200131e

2. World Health Organization. WHO announces COVID-19 outbreak a pandemic, 2020. Available from: http://www.euro.who.int/en/health-topics/health-emergencies/coronavirus-covid19/news/news/2020/3/who-announces-covid-19-outbreak-a-pandemic.

3. World Health Organization. Statement on the second meeting of the International Health Regulations (2005) Emergency Committee regarding the outbreak of novel coronavirus (2019-nCoV). 2020. Available from: https://www.who.int/news/item/30-01-2020-statement-on-the-second-meeting-ofthe-international-health-regulations-(2005)-emergency-committee-regarding-the-outbreak-of-novelcoronavirus-(2019-ncov)

4. World Health Organization. Clinical management of severe acute respiratory infection when Middle East respiratory syndrome coronavirus (MERS-CoV) infection is suspected: interim guidance. 2019. Available from: https://apps.who.int/iris/handle/10665/178529

5. Worldometer. COVID-19 Coronavirus Pandemic. 2020. Available from: https://www.worldometers.info/

6. Sabetian G, Moghadami M, Haghighi LHF, Fallahi MJ, Shahriarirad R, Asmarian N, et al. COVID-19 infection among healthcare workers: a cross-sectional study in southwest Iran. Research Square; 2020.

7. Lotfi M, Sefidbakht S, Moghadami M, Iranpour P, Emami Y, Jafari SH, et al. Introduction of a Radiologic Severity Index for the 2019 Novel Corona Virus (COVID-19). 2020. doi: 10.21203/rs.3.rs47641/v1

8. Shahriarirad R, Sarkari B. COVID-19: clinical or laboratory diagnosis? A matter of debate. Tropical Doctor. 2020. doi: 10.1177/0049475520945446

9. Ashraf MA, Keshavarz P, Hosseinpour P, Erfani A, Roshanshad A, Pourdast A, et al. Coronavirus Disease 2019 (COVID-19): A Systematic Review of Pregnancy and the Possibility of Vertical Transmission. J Reprod Infertil. 2020;21(3):157-68.

10. Mirahmadizadeh A, Ranjbar K, Shahriarirad R, Erfani A, Ghaem H, Jafari K, et al. Evaluation of students' attitude and emotions towards the sudden closure of schools during the COVID-19 pandemic: a cross-sectional study. BMC psychology. 2020;8(1):1-7. doi:10.1186/s40359-020-005007

11. Shahriarirad R, Erfani A, Ranjbar K, Bazrafshan A, Mirahmadizadeh A. The Mental Impact of COVID19 Outbreak: A Population-Based Survey in Iran. 2020. doi: 10.21203/rs.3.rs-23425/v3

12. Erfani A, Shahriarirad R, Ranjbar K, Mirahmadizadeh A, Moghadami M. Knowledge, attitude and practice toward the novel coronavirus (COVID-19) outbreak: a population-based survey in Iran. Bull World Health Organ. 2020. doi: 10.2471/BLT.20.256651 
13. Cheng ZJ, Shan J. 2019 Novel coronavirus: where we are and what we know [published correction appears in Infection. Infection. 2020;48(2):155-163. doi:10.1007/s15010-020-01401-y

14. Young B, Tan TT, Leo YS. The place for remdesivir in COVID-19 treatment. Lancet Infect Dis. 2021;21(1):20-21. doi:10.1016/S1473-3099(20)30911-7

15. Vetter $P$, Kaiser L, Calmy A, Agoritsas $T$, Huttner A. Dexamethasone and remdesivir: finding method in the COVID-19 madness. Lancet Microbe. 2020;1(8):e309-10. doi: 10.1016/S2666-5247(20)30173-7

16. Shahriarirad R, Khodamoradi Z, Erfani A, Hosseinpour H, Ranjbar K, Emami Y, et al. Epidemiological and clinical features of 2019 novel coronavirus diseases (COVID-19) in the South of Iran. BMC infectious diseases. 2020;20(1):1-12. doi: 10.1186/s12879-020-05128-x

17. Huang $C$, Wang $Y, L i X$, et al. Clinical features of patients infected with 2019 novel coronavirus in Wuhan, China. Lancet. 2020;395(10223):497-506. doi:10.1016/S0140-6736(20)30183-5

18. Mehta P, McAuley DF, Brown M, et al. COVID-19: consider cytokine storm syndromes and immunosuppression. Lancet. 2020;395(10229):1033-1034. doi:10.1016/S0140-6736(20)30628-0

19. Stockman LJ, Bellamy R, Garner P. SARS: systematic review of treatment effects. PLoS Med. 2006;3(9):e343. doi:10.1371/journal.pmed.0030343

20. Arabi YM, Mandourah Y, Al-Hameed F, et al. Corticosteroid Therapy for Critically III Patients with Middle East Respiratory Syndrome. Am J Respir Crit Care Med. 2018;197(6):757-767. doi:10.1164/rccm.201706-11720C

21. RECOVERY Collaborative Group, Horby P, Lim WS, et al. Dexamethasone in Hospitalized Patients with Covid-19 - Preliminary Report. N Engl J Med. 2020;NEJMoa2021436. doi:10.1056/NEJMoa2021436

22. Williams DM. Clinical Pharmacology of Corticosteroids. Respir Care. 2018;63(6):655-670. doi: $10.4187 /$ respcare.06314.

23. Sterne J, Murthy S, Diaz J, Slutsky A, Villar J, Angus D, et al. WHO Rapid Evidence Appraisal for COVID-19 Therapies (REACT) Working Group. Association between administration of systemic corticosteroids and mortality among critically ill patients with COVID-19: A meta-analysis. 2020;324(13):1330-41. doi:10.1001/jama.2020.17023

24. Annane D, Pastores SM, Arlt W, Balk RA, Beishuizen A, Briegel J, et al. Critical illness-related corticosteroid insufficiency (CIRCI): a narrative review from a Multispecialty Task Force of the Society of Critical Care Medicine (SCCM) and the European Society of Intensive Care Medicine (ESICM). 2017;43(12):1781-92. doi: 10.1007/s00134-017-4914-x.

25. Hui DS, Sung JJ. Severe acute respiratory syndrome. Chest. 2003;124(1):12-5. doi: 10.1378/chest.124.1.12.

26. Papamanoli A, Yoo J, Grewal P, Predun W, Hotelling J, Jacob R, et al. High-Dose Methylprednisolone in Nonintubated Patients with Severe COVID-19 Pneumonia. 2020:e13458. doi:10.1111/eci.13458

27. World Health Organization. WHO R\&D Blueprint-Novel Coronavirus COVID-19 Therapeutic Trial Synopsis. 2020. Available from: https://www.who.int/publications/i/item/covid-19-therapeutic-trialsynopsis 
28. Association WM. World Medical Association Declaration of Helsinki: Ethical Principles for Medical Research Involving Human Subjects. JAMA. 2013;310(20):2191-4. doi: 10.1001/jama.2013.281053.

29. Meduri GU, Siemieniuk RAC, Ness RA, Seyler SJ. Prolonged low-dose methylprednisolone treatment is highly effective in reducing duration of mechanical ventilation and mortality in patients with ARDS. Journal of Intensive Care. 2018;6(1):53. doi:10.1186/s40560-018-0321-9

30. Braude AC, Rebuck AS. Prednisone and methylprednisolone disposition in the lung. Lancet. 1983 Oct 29;2(8357):995-7. doi: 10.1016/s0140-6736(83)90981-9.

31. Fadel R, Morrison AR, Vahia A, Smith ZR, Chaudhry Z, Bhargava P, Miller J, Kenney RM, Alangaden G, Ramesh MS; Henry Ford COVID-19 Management Task Force. Early Short-Course Corticosteroids in Hospitalized Patients With COVID-19. Clin Infect Dis. 2020;71(16):2114-2120. doi: 10.1093/cid/ciaa601.

32. Alzghari SK, Acuña VSJJoCV. Supportive treatment with tocilizumab for COVID-19: a systematic review. 2020;127:104380. PMID: 32353761

33. Salvi R, Patankar P. Emerging pharmacotherapies for COVID-19. Biomed Pharmacother. 2020;128:110267. doi: 10.1016/j.biopha.2020.110267.

34. Darwish I, Mubareka S, Liles WC. Immunomodulatory therapy for severe influenza. Expert Rev Anti Infect Ther. 2011;9(7):807-22. doi: 10.1586/eri.11.56.

35. Lansbury LE, Rodrigo C, Leonardi-Bee J, Nguyen-Van-Tam J, Shen Lim W. Corticosteroids as Adjunctive Therapy in the Treatment of Influenza: An Updated Cochrane Systematic Review and Meta-analysis. Crit Care Med. 2020;48(2):e98-e106. doi: 10.1097/CCM.0000000000004093.

36. Edalatifard M, Akhtari M, Salehi M, Naderi Z, Jamshidi A, Mostafaei S, et al. Intravenous methylprednisolone pulse as a treatment for hospitalised severe COVID-19 patients: results from a randomised controlled clinical trial. Eur Respir J. 2020;56(6):2002808. doi:10.1183/13993003.02808-2020.

37. Wang Y, Jiang W, He Q, Wang C, Wang B, Zhou P, et al. A retrospective cohort study of methylprednisolone therapy in severe patients with COVID-19 pneumonia. Signal Transduct Target Ther. 2020;5(1):57. doi:10.1038/s41392-020-0158-2

38. Corral L, Bahamonde A, delas Revillas FA, Gomez-Barquero J, Abadia-Otero J, Garcia-lbarbia C, et al. GLUCOCOVID: A controlled trial of methylprednisolone in adults hospitalized with COVID-19 pneumonia. MedRxiv. 2020. doi: 10.1101/2020.06.17.20133579

39. Jeronimo CMP, Farias MEL, Val FFA, Sampaio VS, Alexandre MAA, Melo GC, et al. Methylprednisolone as adjunctive therapy for patients hospitalized with COVID-19 (Metcovid): a randomised, double-blind, phase Ilb, placebo-controlled trial. Clin Infect Dis. 2020;ciaa1177. doi: 10.1093/cid/ciaa1177

40. Nelson BC, Laracy J, Shoucri S, Dietz D, Zucker J, Patel N, et al. Clinical outcomes associated with methylprednisolone in mechanically ventilated patients with COVID-19. Clin Infect Dis. 2020;ciaa1163. doi: 10.1093/cid/ciaa1163 
41. Braude A, Rebuck AJTL. Prednisone and methylprednisolone disposition in the lung. Lancet. 1983;2(8357):995-997. doi: 10.1016/s0140-6736(83)90981-9

42. Hirano T, Homma M, Oka K, Tsushima H, Niitsuma T, Hayashi TJI. Individual variations in lymphocyte-responses to glucocorticoids in patients with bronchial asthma: comparison of potencies for five glucocorticoids. Immunopharmacology. 1998;40(1):57-66. doi: 10.1016/s01623109(98)00025-3.

43. Vichyanond P, Irvin CG, Larsen GL, Szefler SJ, Hill MRJJoa, immunology c. Penetration of corticosteroids into the lung: evidence for a difference between methylprednisolone and prednisolone. J Allergy Clin Immunol. 1989;84(6 Pt 1):867-73. doi: 10.1016/0091-6749(89)90381-3.

\section{Figures}




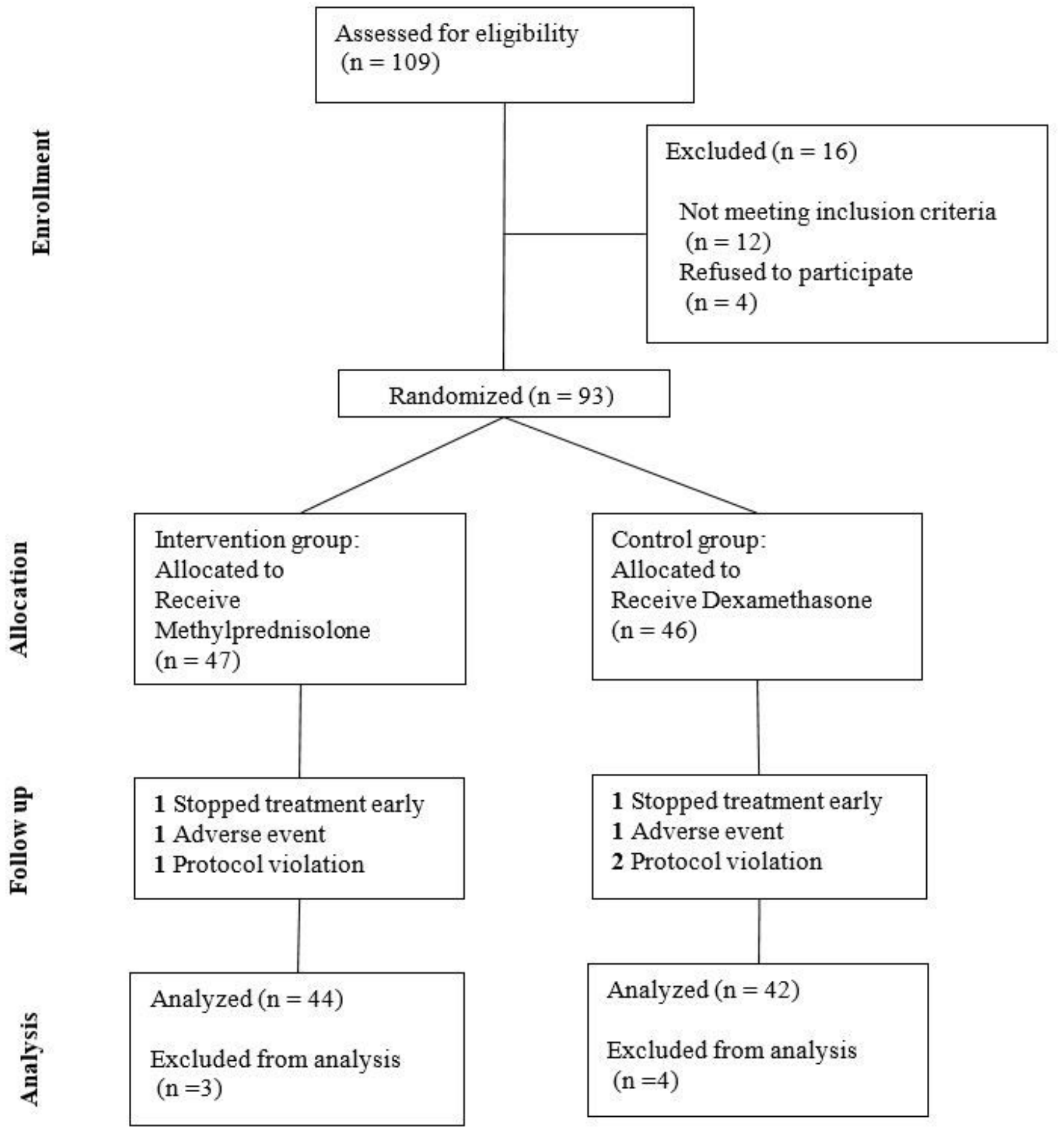

Figure 1

CONSORT Flow diagram of a randomized clinical trial of Methylprednisolone vs. Dexamethasone in patients with COVID-19 


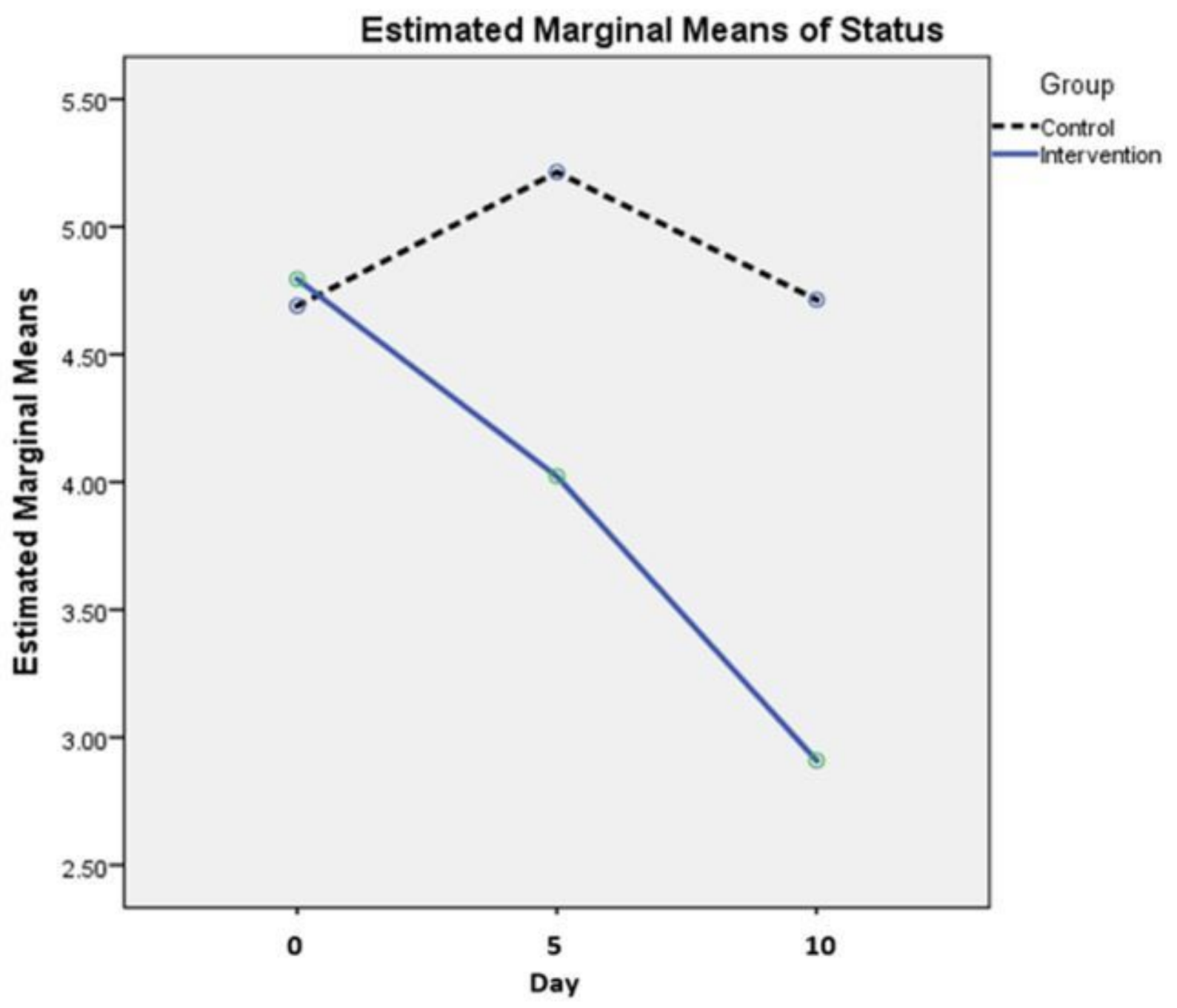

Figure 2

Diagram of clinical status in the intervention (methyl-prednisolone) and control groups 